\title{
Task-specific changes in motor evoked potentials of lower limb muscles after different training interventions
}

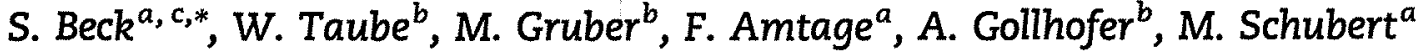 \\ ${ }^{a}$ Department of Clinical Neurology and Neurophysiology, University of Freiburg, Germany \\ bepartment of Sport Science, University of Freiburg, Germany \\ Human Motor Control Section, NINDS, National Institutes of Health, Bethesda, MD, USA
}

A R T I CLE INFO

Keywords:

Training

Plasticity

Motor evoked potentials

Cortico-spinal tract

\begin{abstract}
A B S T R A C T
This study aimed to identify sites and mechanisms of long-term plasticity following lower limb muscle training. Two groups performing either a postural stability maintenance training (SMT) or a ballistic ankle strength training (BST) were compared to a non-training group. The hypothesis was that practicing of a self-initiated voluntary movement would facilitate cortico-spinal projections, while practicing fast automatic adjustments during stabilization of stance would reduce excitatory influence from the primary motor cortex. Training effects were expected to be confined to the practiced task. To test for training specificity, motor evoked potentials (MEP) induced by transcranial magnetic stimulation (TMS) were recorded at rest and during motor tasks that were similar to each training. Intracortical, cortico-spinal, as well as spinal parameters were assessed at rest and during these tasks. The results show high task and training specificity. Training effects were only observable during performance of the trained task. While MEP size was decreased in the SMT group for the trained tasks, MEP recruitment was increased in the BST group in the trained task only. The control group did not show any changes. Background electromyogram levels, M. soleus $\mathrm{H}$-reflex amplitudes and intracortical parameters were unaltered. In summary, it is suggested that the changes of MEP parameters in both training groups, but not in the control group, reflect cortical motor plasticity. While cortico-spinal activation was enhanced in the BST group, SMT may be associated with improved motor control through increased inhibitory trans-cortical effects. Since spinal excitability remained unaltered, changes most likely occur on the supraspinal level.
\end{abstract}

1.

\section{Introduction}

Control of leg movements, such as balance and gait, plays an important role in human everyday life. A great number of empiric training interventions have been developed for rehabilitation purposes and in sports science in order to improve postural control and leg motor performance. The acquisition of motor skills in the first 4 to 6 weeks of training primarily relies on neural plasticity rather than on changes in muscle structure (Enoka, 1997; Komi, 1986; Ploutz et al., 1994). With respect to lesion sites within the central nervous system (e.g. spinal or cortical), it is important to understand whether a

\footnotetext{
* Corresponding author. Human Motor Control Section, NINDS, National Institutes of Health, Bldg. 10/5N240, 10 Center Drive, Bethesda, MD 20892-1428, USA. Fax: +1 3014802286.

E-mail address: becksa@ninds.nih.gov (S. Beck).
} 
particular training preferentially induces plastic changes in spinal or cortical sensory-motor circuits. It is also crucial to know under which conditions this can best be observed.

So far, most studies were focused on acute training-induced effects, exploring just minutes to hours after a training intervention. For such immediate effects, transcranial magnetic stimulation (TMS) studies identified predominantly cortical plasticity such as increased motor cortex excitability and enlargement of motor cortical representation (Classen et al., 1998; Pascual-Leone et al., 1994, 1995). Analogous to hand function, gait and postural control are highly adaptable and underlie specific cortical control (Bonnard et al., 2002; Camus et al., 2004; Christensen et al., 2001; Schubert et al., 1999; Taube et al., 2006). Likewise, short-term motor skill training in the leg muscles induces increased motor cortex excitability, which is consistent with findings in the upper limb suggesting a similar underlying principle (Perez et al., 2004).

In contrast to acute adaptation, the effects of long-term execution of specific motor interventions are less well known and only a few electrophysiological studies have addressed this issue so far (Carroll et al., 2002; Jensen et al., 2005; Taube et al., 2007). While functional brain imaging studies have shown that the main focus of activity shifts from cortical towards subcortical motor regions including cerebellar dentate nucleus, thalamus and putamen during the time course of training (Doyon and Benali, 2005; Floyer-Lea and Matthews, 2004; Wu et al., 2004), they did not assess the role of spinal plasticity. Findings from TMS experiments have shown a decrease in cortico-spinal excitability correspondingly. This was a common finding in two studies on strength training of upper limb muscles (Carroll et al., 2002; Jensen et al., 2005). However, the motor conditions under which this was detected were not really comparable. While changes were exclusively reported at rest in one study (Jensen et al., 2005), they were observed only during tonic activation in the other (Carroll et al., 2002). Still -another recent study on balance training showed a selective reduction of cortico-spinal excitability only during perturbation of stance, a task resembling the one which was performed during the training (Taube et al., 2007). Thus, apparently the motor condition under which training effects are assessed may be as influential for the result as the training itself. Furthermore, it has been shown that different motor training regimen induce disparate forms of neurophysiologic changes in different anatomic motor areas (Adkins et al., 2006; Jensen et al., 2005).

The goal of this study was to identify sites and mechanisms of long-term plasticity following lower limb muscle training by comparing two different training interventions. Both training regimens are widely used for athletic training and in rehabilitation. They have a similar effect on developing explosive muscle strength (Gruber et al., 2007a; Taube et al., 2007). However, the build-up of rapid force increment (i.e. explosive strength) is attained in different ways. While postural stability maintenance training (SMT) consists of fast automatic movements, leaving no time to consider or prepare the required move, ballistic ankle strength training (BST) strongly emphasizes mental preparation preceding each move to deliberately initiate muscle contraction. It was hypothesized that cortical plasticity is involved in long-term training and that training effects would best be observable in the corresponding active state as training is known to be rather specific for the particular trained task (Voigt et al., 1998). Therefore, cortico-spinal (motor evoked potential (MEP) size and MEP recruitment), intracortical (short intracortical inhibition (SICI) and intracortical facilitation (ICF)) and spinal (M. soleus H-reflex) measures were taken at rest and during motor tasks similar to each type of training. MEP recruitment was expected to be enhanced after BST paralleling the known increase of EMG and force development (Aagaard et al., 2002; Adkins et al., 2006). Cortical excitability was assumed to be reduced after SMT, as shown in a current study (Taube et al., 2007). A control group also underwent testing in order to distinguish training-specific effects from task-induced activation. Better knowledge about specific and generalized effects induced by each type of training may allow goal-oriented application and thereby improve motor outcome.

\section{Results}

\subsection{Background EMG}

Amplitudes or force levels for all movements were individually adjusted before training to evoke similar levels and slopes at the first peak of EMG (see Fig. 1). The latency of EMG onset and the first peak of EMG in $\mathrm{M}$. tibialis anterior and $\mathrm{M}$. soleus, representing the point in the time course of movement, at which electrical and magnetic stimulation were applied, were neither different before and after training nor between groups.

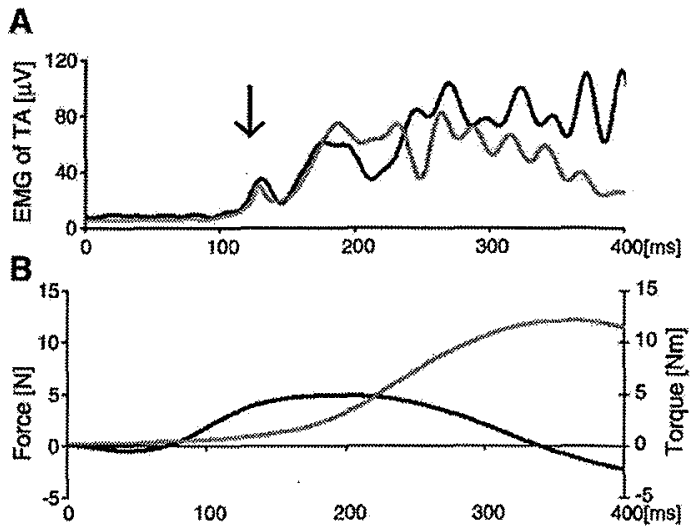

Fig. 1 - Time course of EMG and force levels during dorsiflexion and forward perturbation in $\mathrm{M}$. tibialis anterior. (A) The time course of EMG level in M. tibialis anterior (rectified and filtered average of ten trials) before training in one subject is shown for both tasks, in which M. tibialis anterior is the agonist (DFL (gray) and PERf (black)). Stimulation parameters (force and amplitude for perturbations) were adjusted to evoke similar EMG levels in the first phase of the contraction (indicated by the black arrow (l)) for both motor tasks in order to attain similar activation levels in the alpha-motoneuron pool. (B) The time course of the differential force during PERf on the treadmill (gray, left ordinate) and of the torque measured by the force transducer in the footplate during DFL (black, right ordinate) is shown. 
Motor tasks, in which the muscles were targeted (DFL and PERf for $M$. tibialis anterior, PFL and PERb for M. soleus), led to higher EMG levels compared to movements in the opposite direction (ANOVA $p<0.001$ ), however, there was no difference in EMG levels before and after training (ANOVA n.s.) (see Table 1).

\subsection{M. soleus H-reflex}

Maximal M. soleus $H$-reflex amplitude was expressed as percentage of maximal $\mathrm{M}$-wave $\left(\mathrm{H}_{\max } / \mathrm{M}_{\max }\right.$ ratio). Changes in the $H_{\max } / M_{\max }$ ratio are assumed to reflect changes in spinal excitability if afferent input is controlled (Zehr, 2002). Independent from the type of training, $H_{\max } / M_{\max }$ ratio was significantly modulated by the motor tasks (ANOVA $p<0.001)$. It was increased during tasks in which $M$. soleus was activated (PFL $H_{\max } / M_{\max }=64.1 \pm 1.9 \%$; PERb $H_{\max } /$ $\left.M_{\max }=60.3 \pm 2.1 \%\right)$ compared to rest $\left(H_{\max } / M_{\max }=54.4 \pm 2.7 \%\right)$. This pattern of task-related M. soleus H-reflex modulation was similar in all three groups and was preserved after training. After the intervention, the difference in $H_{\max } / \mathrm{M}_{\max }$ per group and task was: SMT $-2.8 \pm 3.7 \%$ in PFL,$-5.3 \pm 3.7 \%$ in PERb, $3.9 \pm 5.3 \%$ in R; BST $4.2 \pm 2.5 \%$ in PFL, $2.3 \pm 3.4 \%$ in PERb, $8.7 \pm 5.0 \%$ in $R$ (ANOVA n.s.).

$M_{\max }$ was not different between motor tasks, groups or before and after training $\left(M_{\max }(\right.$ pre $)=5.9 \pm 0.2 \mathrm{mV} ; M_{\max }$ (post) $=$ $5.8 \pm 0.2 \mathrm{mV}$; ANOVA n.s.).

\subsection{Motor threshold}

There was no change in MT in any of the three groups (MT (pre) $=59.8 \pm 1.7 \%$ maximum stimulator output intensity (MSI); MT (post) $=59.4 \pm 1.8 \%$ MSI (ANOVA n.s.)).

\subsection{Short intracortical inhibition and intracortical facilitation}

Conditioning of a suprathreshold TMS stimulus by a preceding subthreshold stimulus led to an inhibition at an interstimulus interval of $2 \mathrm{~ms}$ and $5 \mathrm{~ms}$ and to a facilitation at an inter-stimulus interval of $13 \mathrm{~ms}$ and $15 \mathrm{~ms}$. The mean MEP size

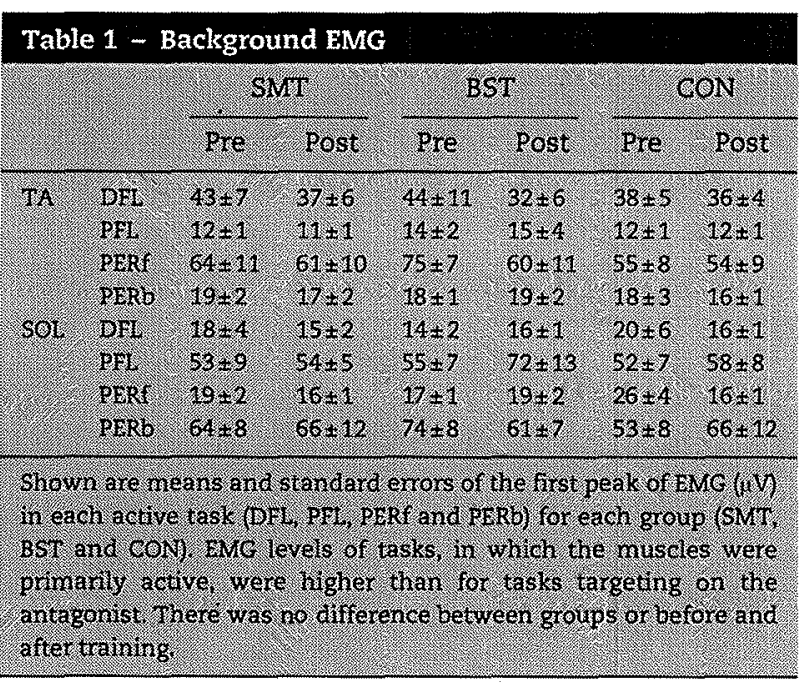

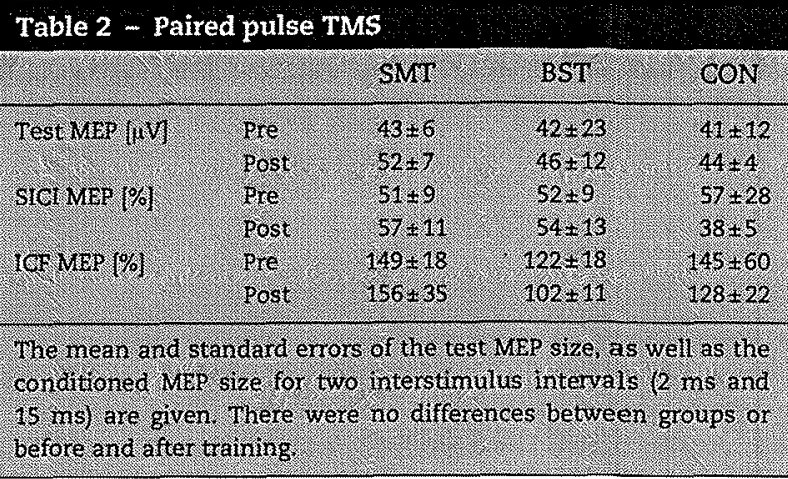

of the test stimulus was $48 \pm 29 \mu \mathrm{V}$ and did neither differ between the training groups, nor change before and after training (ANOVA n.s., see Table 2).

The amount of inhibition and facilitation was different depending on the inter-stimulus interval (ANOVA $p<0.001$ ), while there was no difference between training groups and between the pre- and post-measurement (see Table 2).

\subsection{MEP size at rest and during motor tasks}

After the four week period of exercise, there was no significant overall effect of training on MEP size of M. tibialis anterior or M. soleus, when comparing the changes calculated as difference between pre-training and post-training assessment (see Experimental procedures). However, there was a significant task effect on absolute MEP size in M. tibialis anterior prior to any training; that is, $M$. tibialis anterior MEP size was modulated differently throughout the five tasks (ANOVA "task", $p<0.001$ ). The interaction of "task" and "training" did not reveal significant differences (ANOVA "task" "training" $p=0.084$ ). If the control group was excluded from the analysis, this interaction of task and training was significant (ANOVA

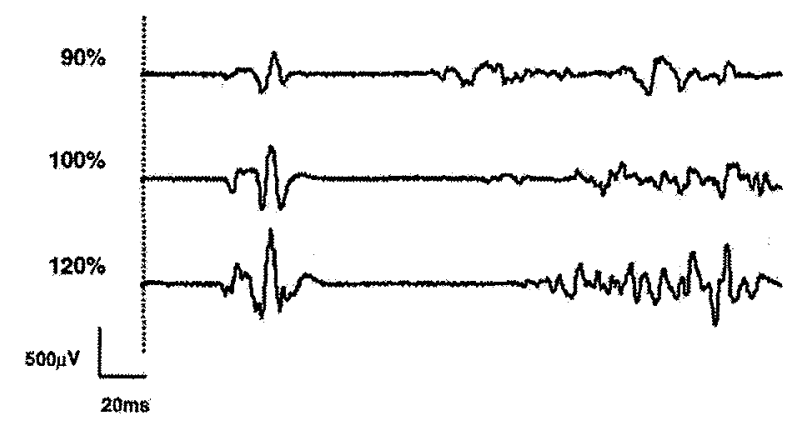

Fig. 2 - MEP size for the different stimulation intensities during motor activation. Single MEPs in one subject are shown for the three different stimulation intensities applied $(90 \% \mathrm{MT}, 100 \% \mathrm{MT}$ and $120 \% \mathrm{MT})$ in M. tibialis anterior during DFL. The dotted line represents the moment, when the cortical stimulation (TMS) was given. The stimulation was timed to evoke an MEP during the first peak of EMG and induced a silent period (interruption of background EMG) of increasing duration when the stimulus intensity was enhanced. 
$p<0.05$ ). Accordingly, the one sample analysis revealed a decrease in $\mathrm{M}$. tibialis anterior MEP size during tasks which were similar to the training in the SMT group. Effects were significant for both perturbation tasks (PERf: $\triangle \mathrm{MEP}=-78 \pm$ $24 \mu \mathrm{V} ; p<0.05$; PERb: $\triangle \mathrm{MEP}=-43 \pm 11 \mu \mathrm{V} ; p<0.01$; see Fig. 3). In the BST group, $M$. tibialis anterior MEP size was reduced during PFL ( $\triangle M E P=-71 \pm 28 \mu V ; p<0.05$ ). There was a trend for an increase in M. tibialis anterior MEP size during DFL ( $\triangle \mathrm{MEP}=92 \pm$ $42 \mu \mathrm{V} ; p=0.067$; see Fig. 3).

MEP size in $M$. soleus was not modified after any type of training (ANOVA "task" n.s.). There was no significant interaction of task and training (ANOVA "task" "training" n.s.). The one sample analysis showed no significant group effect in any task (see Fig. 3). No training-induced changes were detected at rest.

\subsection{MEP recruitment at rest and during motor tasks}

The application of TMS pulses of $90 \% \mathrm{MT}$ at rest induced MEPs with a mean size of $14.2 \pm 0.1 \mu \mathrm{V}$ before training and $12.3 \pm$ $0.1 \mu \mathrm{V}$ after training, respectively, which was not larger than background EMG activity. During activation by the tasks, the $90 \% \mathrm{MT}$ stimulation intensity led to reproducible MEPs in all subjects (see Fig. 2) and therefore all three values were used for the linear fit.

A
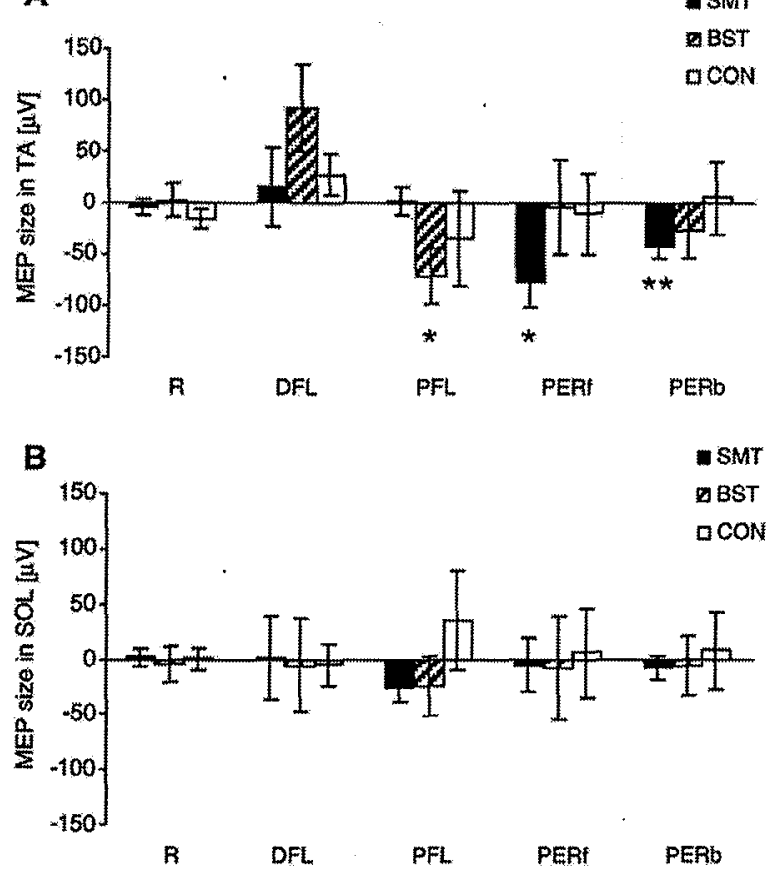

Fig. 3 - MEP size. Shown is the mean of the individual difference in MEP size before and after training in $M$. tibialis anterior (A) and M. soleus (B) for all treatments (SMT (black), BST (striped) and CON (white)). The stimulation intensity was $120 \%$ MT for all tasks. MEP size was reduced in TA during both perturbations (as the trained movements) after SMT and for PFL after BST. There was a trend for an increase of MEP size in M. tibialis anterior during DFL after BST (the trained task). No modulation was observed in M. soleus. Stars indicate the level of significance $\left({ }^{*} p<0.05,{ }^{* *} p<0.01,{ }^{* * *} p<0.001\right)$.

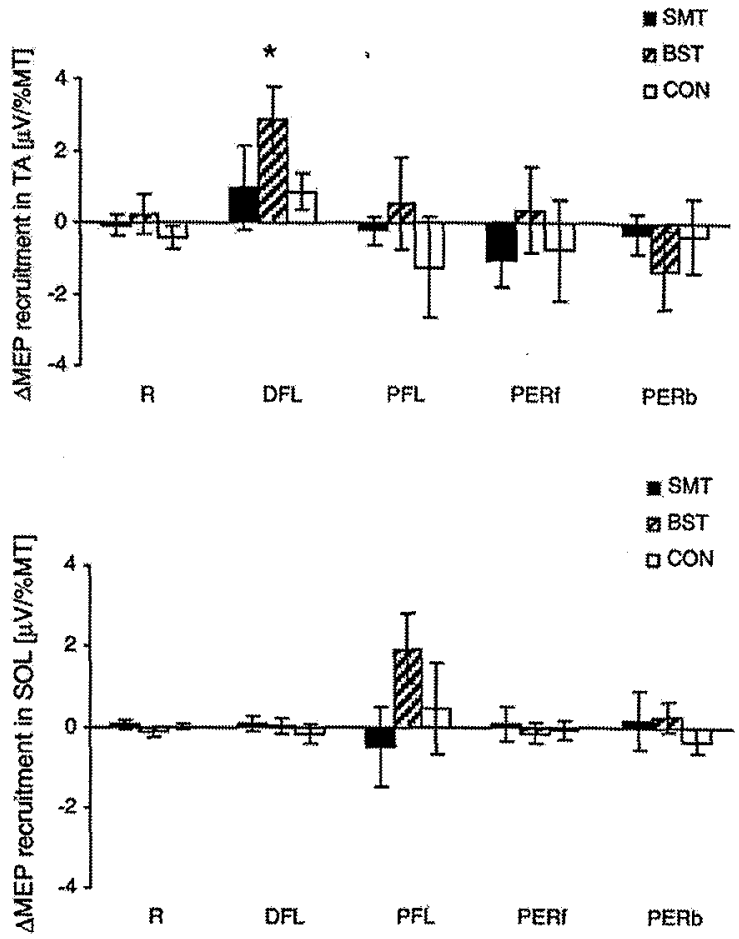

Fig. 4 - MEP recruitment. Shown are mean and standard errors of the individual difference in MEP recruitment before and after training in $M$. tibialis anterior $(A)$ and $M$. soleus (B) for all treatments (SMT (black), BST (striped) and CON (white)). The results show a highly task- and trainingspecific change in MEP recruitment. While there was no modulation for the SMT group or CON, MEP recruitment increased during DFL in $M$. tibialis anterior (trained movement) and showed a trend to increase during PFL in SOL (trained movement). Stars indicate the level of significance $\left({ }^{*} p<0.05,{ }^{* *} p<0.01,{ }^{* * *} p<0.001\right)$.

Analogous to MEP size, there was no significant overall training effect on $M$. tibialis anterior or $M$. soleus MEP recruitment changes. However, even prior to any training, there was also a significant task effect on $M$. tibialis anterior MEP recruitment (ANOVA "task" $p<0.05$ ). The interaction of task and training was not significant (ANOVA "task" * "training" n.s.). Despite this, the one sample analysis showed an increase in $M$. tibialis anterior MEP recruitment in the trained task after BST (DFL; $\triangle \mathrm{MEP}$ recruitment $=2.86 \pm 1 \mu \mathrm{V} / \% \mathrm{MSI} ; p<0.05$; see Fig. 4).

For M. soleus recruitment, there were no significant task effect and no interaction for task and training when comparing all three groups. When only the two trainings were compared, an interaction of task and training was obvious (ANOVA "task" "training" $p<0.05$ ). The one sample analysis revealed a trend for an increase in $M$. soleus MEP recruitment during the trained task in the BST group ( $\triangle \mathrm{MEP}$ recruitment $=1.94 \pm 1 \mu \mathrm{V} / \% \mathrm{MSI} ; p=0.062$; see Fig. 4).

No significant modulation of MEP recruitment was observed after SMT in any of the tasks. No changes were detected at rest. 


\section{Discussion}

The purpose of this study was to assess adaptive changes after long-term training of lower limb muscles. In order to identify the sites and specificity of induced plasticity, two different well-established training regimens were studied. While both trainings are known to increase explosive muscle strength (Gruber et al., 2007a,b; Taube et al., 2007), the mechanisms were expected to be different. Postural stability maintenance training (SMT, termed sensorimotor training in the former studies) consists of fast, automatic ankle movements in response to postural imbalance with the goal of stabilizing the body on the unstable training device. In contrast, ballistic ankle strength training (BST) strongly emphasizes mental preparation preceding each voluntary contraction. Therefore, a reduction of cortico-spinal excitability was expected in the SMT group, in contrast to an enhancement in the BST group. These effects were hypothesized to be specific for the particular training task. Spinal, cortico-spinal and intracortical parameters were assessed at rest and during active tasks that were similar to the trainings. In accordance with the hypothesis, a task- and training-specific reduction of MEP size was observed in the SMT group for the trained task only, while a task- and training-specific enhancement of MEP recruitment was found in the BST group. These findings underline previous results in that training is effective for the trained task and that it plays an important role under which conditions electrophysiological assessment is performed. The lack of modulation of spinal excitability suggests that relevant changes most likely occur on the cortical level.

3.1. The role of primary motor cortex during short- and long-term training

Generally, brain imaging studies as well as electrophysiological studies have provided strong evidence for a crucial role of the primary motor cortex during the early phase of any type of motor skill acquisition (Butefisch et al., 2004; Classen et al., 1998; Doyon and Benali, 2005; Lotze et al., 2003; Muellbacher et al., 2001, 2002; Pascual-Leone et al., 1994, 1995; Puttemans et al., 2005). Despite few contradictory results (Bonato et al., 2002, 1994), increased cortical excitability during early motor skill acquisition is well documented for the upper limb (PascualLeone et al., 1994, 1995). Similarly, increased recruitment of TMS-induced MEPs has been observed directly after skill training for lower limb muscles suggesting similar mechanisms (Perez et al., 2004). This finding was underlined by the observation of a significant increase of EEG-EMG coherence suggesting that the modulation of cortico-spinal drive to spinal motoneurons was relevant for motor learning (Perez et al., 2006).

Excitability changes within the cortico-spinal system can be observed after several weeks of training. This is one common finding of former long-term training studies, although the motor conditions under which they were observed were not uniform (Carroll et al., 2002; Jensen et al., 2005; Taube et al., 2007). While enhancement of maximum MEP size and lowered motor threshold after motor skill training were demonstrated during tonic contraction, strength training was shown to decrease TMS-induced MEP recruitment at rest (Taube et al., 2007). In contrast, Carroll et al. (2002) observed a reduction of TMS and transcranial electrical stimulation (TES) induced recruitment curves after strength training under tonic activation only and assumed a subcortical site of plasticity. Such contradictory results on the involvement of the motor cortex may well be due to differences in motor tasks which were performed by the subjects while neurophysiologic measures were recorded. In a current study, it has been demonstrated that long-term sensorimotor training decreases cortico-spinal excitability during body perturbations (Taube et al., 2007). Using a TMS conditioning technique, the authors were able to demonstrate a modulation of cortical influence by comparing short and long latency reflexes in a perturbation task similar to the trained movement. This may indicate a crucial influence of the motor condition on neurophysiologic assessment of training effects, as previously assumed (Enoka, 1997).

3.1.1. The role of task specificity during measurement of changes in cortical excitability

As training is usually specific for the type of the practiced movement (Aagaard et al., 2002; Enoka, 1997; Voigt et al., 1998), the present investigation extends previous findings and demonstrates that, in order to detect training adaptations by electrophysiological tests, the motor task used for the testing must be considered. As assumed from the former studies, our results prove a crucial influence of a motor condition on training effects. If this is taken into consideration, specific training effects may be observable provided that the training and motor conditions match. In our study, this was the case for the combination of BST with plantarflexion/dorsoflexion and of SMT with forward/backward perturbation. These were the only combinations of test conditions where effects of SMT and BST were detectable, as indicated by the significant interaction effects of task and training. No training effects were detectable during similar, untrained movements with comparable force levels and joint angles, e.g. when testing MEP during perturbation in the BST group (PERf and PERb, Fig. 3) or during dorsiand plantarflexion in the SMT group (DFL and PFL, Figs. 3 and 4). Hence, adaptations were specific for the trained task after both interventions.

\subsubsection{Differences in cortical excitability are related to training} task specificity

These results are at difference with previous findings of decreased MEP recruitment following strength training (Carroll et al., 2002; Jensen et al., 2005). This may be due to a difference in the training protocols, which were not focused on explosive strength in the former studies. Furthermore a difference of target limbs and, as pointed out before, an incongruous combination of training and testing conditions may account for this discrepancy and, thus, for the failure of the former studies to detect an increase in recruitment.

Contrary to these inconsistencies, an important finding of the present study is the task-specific reduction of MEP size in the SMT group in the trained tasks, which is in line with another study addressing long-term effects of this type of training in the lower limb (Taube et al., 2007). Similar to the observations from the BST group, adaptation was only detectable during perturbations, which matched the movements during SMT. The interpretation 
of this result is more complex. Given that spinal excitability remained largely unchanged (as is suggested by unaltered $\mathrm{H}$ reflex tests), a supraspinal site of plasticity may be considered for this type of training as well. Inhibition is a very common cortical principle to regulate subsidiary neural circuits. The reduction of MEP size may therefore reflect an increase of cortical inhibitory control of alpha-motoneurons, e.g. via increase of presynaptic inhibition (Gruber et al., 2007a; Taube et al., 2007). Interestingly, MEP size in $M$. tibialis anterior was also reduced in a trained task in the BST group, but in this case the reduction was observed during the activation of the antagonist. This may suggest a more selective control of the antagonists as a result of the training.

\subsection{Putative mechanisms and methodological limitations}

In general, the lacking overall group effect for comparisons between SMT, BST, and CON may be suspect of underpowered statistical testing. This is possible as numbers of included subjects per group were rather low. However, even these numbers were sufficient to clearly show strong interaction effects and demonstrate the strong influence of motor task on observable training effects. Under these circumstances, the current study shows that MEP recruitment in the BST group is increased after the training period. MEP recruitment evaluates input-output properties of the motor system (Chen et al., 1998). It reflects excitatory synaptic transmission in the motor cortex and density of corticospinal projections to the muscle assessed (Chen et al., 1998). Yet, it may also be modulated by differences in the recruitment order of spinal alpha-motoneurons (Devanne et al., 1997). The latter mechanism could not be verified by H-reflex testing. Therefore, this may suggest that these changes were largely cortical in origin.

Several studies support the view that $\mathrm{GABA}^{\mathrm{A}}$-mediated SICI plays an important role in plasticity of the primary motor cortex Jacobs and Donoghue, 1991; Liepert et al., 1998; Pascual-Leone et al., 1995; Schneider et al., 2002; Werhahn et al., 1999). In the current study, SICI and ICF remained unchanged after training. However, intracortical tests were only performed at the end of the four-week training period. Intracortical training effects occurring early during training therefore may have been missed. Thus, it must be conceded that lack of modulation in SICI is not contradictory to the concept derived from fMRI studies (Doyon and Benali, 2005), which shows that acute changes are accompanied by an increase of activity at the cortical level, which shifts towards subcortical sites during the process of motor learning. Moreover, SICI and ICF were only assessed at rest. Therefore, intracortical changes in the active state cannot be ruled out.

In summary, we conclude that supraspinal sites are involved in long-term adaptation after explosive type of leg muscle training. Probably motor cortex is involved, as was hypothesized and could be demonstrated by electrophysiological assessment. Hence, such trainings are capable to alter cortico-spinal facilitation, possibly by changing recruitment gain. However, the exact mechanism of modulation in recruitment gain after BST remains unclear. Notably, adaptations were task-specific and could not be seen at rest or in non-trained movements. Thus the congruence of motor task during training and during electrophysiological assessment proves to be relevant to detect specific training effects.

\section{Experimental procedure}

\subsection{Participants}

Twenty-seven healthy subjects aged $20-38$ years (ten females, seventeen males) participated in this study. Participants gave their informed consent to the experiments, which were approved by the local ethics committee according to the Declaration of Helsinki. Care was taken to screen subjects for any medication usage with potential effects on cortical excitability, history of seizures, neurosurgery, metal implants or injuries affecting the ankle joint. Participants were randomly allocated to one of three treatment groups: postural stability maintenance training (SMT, $n=9$ ), ballistic ankle strength training (BST, $n=8$ ) and a control group (CON, $n=10$ ) (see Table 3).

\subsection{Training}

Both training groups (SMT and BST) pursued a specific training protocol over a four-week time period, with a total of sixteen training sessions, while CON maintained their average amount of physical activity. All training sessions were documented, surveyed and supervised by the authors of the study. Each session lasted for $60 \mathrm{~min}$ starting with a $10 \mathrm{~min}$ warm-up program on a bicycle dynamometer and ending with a $10 \mathrm{~min}$ cool-down program on a bicycle dynamometer.

SMT consisted of four postural stabilization tasks including a wobbling board, a spinning top, a soft mat (Airex, Aalen, Germany) and a balance pad. Each task was performed unilaterally (right leg) with slightly flexed knee while looking straight ahead and hands held akimbo. The first goal was to stay in an upright position on the training device. When subjects had learned to stand on the device for the whole time period, they were instructed to minimize body sway. The difficulty of the devices varied in that it was possible for all subjects to stabilize themselves on the soft mat from the beginning, while not all of them were able to stay on the spinning top for $20 \mathrm{~s}$, even at the end of the training protocol.

In one training session, all four devices were used in a randomized order. One set of exercise with one postural

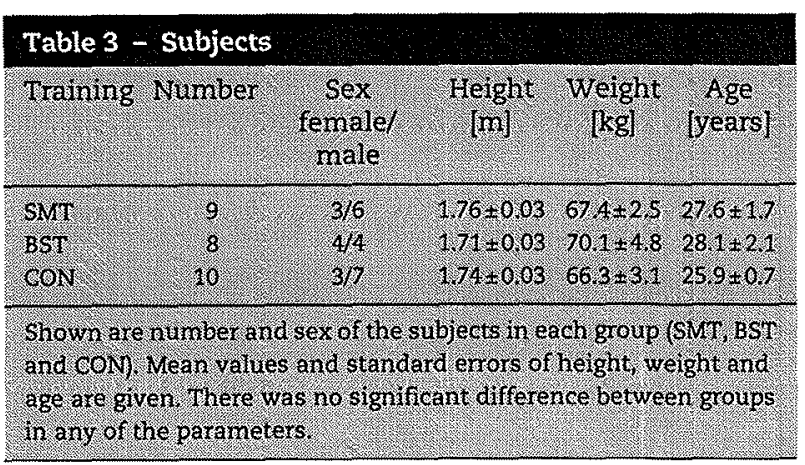


training device consisted of four periods of $20 \mathrm{~s}$ of exercise alternated with $40 \mathrm{~s}$ rest. In order to avoid fatigue a resting time of $3 \mathrm{~min}$ was scheduled prior to the next device. After four training sessions, the number of sets performed on each device was increased to six. Difficulty was increased during the last four training sessions by performance with eyes closed. This training regimen had been described in detail by Gruber et al. (2007a).

BST was performed according to established protocols (Duchateau and Hainaut, 1984; Schmidtbleicher and Haralambie, 1981; Van Cutsem et al., 1998). The subjects were positioned with the right ankle joint at $100^{\circ}$. In each training session, subjects executed four sets of 10 dorsal flexions and 10 plantar flexions. The contractions were performed self-initiated and at maximum velocity against a load of 30 to $40 \%$ of their one repetition maximum. The main goal was to exert the required force as fast as possible. Between contractions, subjects rested for $2 \mathrm{~s}$. The rest period between the sets was $3 \mathrm{~min}$ in order to avoid fatigue, similarly to SMT. Training load was adjusted after 2 weeks to consider training effects. Like in the SMT protocol, training was intensified after four sessions by increasing the number of sets to six and difficulty was increased during the last four sessions by performing the training with eyes closed.

\subsection{Testing before and after training}

All subjects underwent all tests. Efficiency of training was verified by a significant increase of maximum explosive strength (rate of force development) and stance stability, as was shown in earlier studies (Gruber et al., 2007a,b; Taube et al., 2007). Electrophysiological assessment was performed at rest and during specifically designed motor tasks mimicking the trained movements. Before and after training, the experiments were performed in the same order. The final assessment was conducted between 24 and $72 \mathrm{~h}$ after the last training session.

\subsubsection{Procedures}

4.3.1.1. EMG recording. Bipolar $\mathrm{Ag}-\mathrm{AgCl}$ surface electrodes ( $9 \mathrm{~mm}$ diameter, $3 \mathrm{~cm}$ distance between poles) were placed over the belly and tendon of three muscles of the right leg (M. tibialis anterior, M. gastrocnemius and M. soleus). The cup electrodes were filled with electrode jelly and after skin preparation firmly attached to the skin by means of doublesided adhesive tape. Impedance was reduced below $5 \mathrm{k} \Omega$. EMG signals and ground reaction forces were sampled at $4 \mathrm{kHz}$, amplified and bandpass filtered $(30-2000 \mathrm{~Hz})$.

At the beginning of the electrophysiological assessment subjects walked on the treadmill with a velocity of $1.1 \mathrm{~m} / \mathrm{s}$ and EMG amplitudes for $M$. tibialis anterior and $M$. soleus were assessed by averaging at least fifty steps. The first peak EMG levels of all motor tasks (dorsoflexion, plantar flexion and perturbations; see below) were matched to the level of the first peak in EMG during gait in M. tibialis anterior and $M$. soleus by adjusting the amplitude of perturbation. The rational was to receive comparable background levels of activation in the target muscle in order to ensure a similar level of alpha-motoneuron excitability in all motor conditions tested (see Fig. 1). The adjustment to gait was conducted to assess an average level of motor activation and force production, which was assumed more relevant for daily behavior than maximum force levels.

For each task, EMG of at least ten trials was recorded and averaged. Then the latency for the first peak of the EMG was individually determined. This latency was chosen to time the stimulation (peripheral nerve stimulation and TMS). EMG amplitude was determined by calculating the root mean square of the first EMG peak (from $50 \mathrm{~ms}$ before until $50 \mathrm{~ms}$ after the peak) in each muscle. For M. tibialis anterior, this value was normalized to the EMG at heel strike during gait (from $50 \mathrm{~ms}$ before until $50 \mathrm{~ms}$ after heel strike, calculated as RMS). For M. soleus, the value obtained was normalized on $M_{\max }$ obtained during $M$. soleus $H$-reflex assessment. During perturbation and gait, downward directed force and anteroposterior directed differential force were recorded.

4.3.1.2. Electrical stimulation. For peripheral nerve stimulation, an electrical stimulator (Digitimer ${ }^{\oplus}$ DS 7, Hertfordshire, UK) was used to generate single square-wave pulses of $500 \mu \mathrm{s}$ duration. The cathode ( $2 \mathrm{~cm}$ in diameter) was placed over the tibial nerve in the popliteal fossa and the anode $(10 \mathrm{~cm} \times 5 \mathrm{~cm})$ was positioned below the patella. M. soleus H-reflex recruitment curves were recorded at rest and during plantarflexion (PFL) and backward perturbation (PERb) for M. soleus. The electrical stimulation was timed to evoke an $\mathrm{H}$-reflex at the first peak of EMG. Recruitment curves were displayed online (LabView, National Instruments, Texas USA). For each subject the maximal amplitude of the $M$-response $\left(M_{\max }\right)$ and the maximal amplitude of $\mathrm{H}$-reflex $\left(\mathrm{H}_{\mathrm{max}}\right)$ were determined and the $H_{\max } / M_{\max }$ ratio was calculated in order to compare changes in spinal excitability between different motor conditions (Zehr, 2002).

4.3.1.3. TMS. TMS was applied by using two commercially available stimulators (MagPro 200, Magstim Company, Dyfed, UK). The stimulus waveform was monophasic. A circular coil (diameter $90 \mathrm{~mm}$ ) was used with the current flowing counterclockwise. At the beginning of each experiment (before and after training) the motor hot spot for $\mathrm{M}$. tibialis anterior was determined by systematically changing the position of the coil over the primary motor cortex. The coil was held tangentially to the skull. At the optimal position, the center of the coil, which was usually $0-1 \mathrm{~cm}$ anterior and $0-2 \mathrm{~cm}$ lateral $\mathrm{Cz}$, and the inner and outer circumference of the coil were marked in order to keep the coil position constant during all experiments. $M$. tibialis anterior was selected as a target muscle for the placement and determination of resting motor threshold because MEPs were easily evoked at rest, whereas this was not possible for M. soleus in all subjects. Motor threshold (MT) for $M$. tibialis anterior was individually determined as the minimal stimulus intensity required to evoke MEPs with amplitudes larger than $100 \mu \mathrm{V}$ in at least three out of five consecutive trials. Motor threshold was expressed in percentage of the maximum stimulator output intensity (MSI). In the sitting position, the head of the subject was fixed tightly to a headrest on which the coil was attached. During gait and 
perturbations, subjects wore a harness introduced by Schubert et al. (1997) with a dorsal headrest affixed very tightly in order to keep the position of the coil constant throughout the experiments. Between tests, the position of the coil was controlled visually and by application of defined stimuli.

MEP amplitudes were assessed at rest and in the active state by applying at least ten TMS pulses of $90 \% \mathrm{MT}, 100 \%$ MT and $120 \%$ MT. Since the stimulation intensity needed to evoke MEPs in leg muscles is generally higher compared to upper limb muscles (mean values reported are $60-70 \%$ of maximum stimulator output), these stimulation intensities were selected in order to be able to test them in all subjects and to avoid saturation effects during activity. Under activity, all three stimulation intensities were suprathreshold and consequently evoked reproducible MEPs in both muscles (see Fig. 2). Root mean square (RMS) values were determined over $30 \mathrm{~ms}$ after the individual onset of MEP. At-rest trials with EMG values (RMS) above $20 \mu \mathrm{V} 15 \mathrm{~ms}$ prior to MEP were rejected. To assess changes in MEP size, MEP amplitudes with a stimulation intensity of $120 \%$ MT were compared between tasks. MEP recruitment was determined by calculating the slope of a linear fit of all three values. It has been shown that this is an appropriate way to analyze MEP recruitment and produces comparable results to complex non-linear fits (Darling et al., 2006).

$\mathrm{SICI}$ and ICF were determined for $\mathrm{M}$. tibialis anterior at rest by applying a preceding conditioning stimulus of $80 \% \mathrm{MT}$ followed by a suprathreshold test stimulus of $120 \% \mathrm{MT}$ (Kujirai et al., 1993). Ten test pulses and ten conditioned pulses of each inter-stimulus interval $(2 \mathrm{~ms}, 5 \mathrm{~ms}, 8 \mathrm{~ms}$, $13 \mathrm{~ms}$ and $15 \mathrm{~ms}$ ) were applied in a randomized order. RMS values over $30 \mathrm{~ms}$ after the individual onset of the MEP were calculated and trials with EMG values (RMS) above $20 \mu \mathrm{V}$ $15 \mathrm{~ms}$ prior to MEP were rejected. While interstimulus intervals of $2 \mathrm{~ms}$ and $5 \mathrm{~ms}$ usually lead to a reduction of MEP size, $13 \mathrm{~ms}$ and $15 \mathrm{~ms}$ are known to facilitate the test MEP amplitude (Kujirai et al., 1993).

\subsubsection{Motor tasks for the electrophysiological assessment}

4.3.2.1. Ankle perturbation tests at rest and similar to SMT. In a sitting position (on a chair; starting position for the movement: hip flexion $110^{\circ}$, knee $120^{\circ}$, ankle joint $100^{\circ}$ ) three different tasks were assessed: rest $(\mathrm{R})$, dynamic dorsoflexion (DFL) and dynamic plantar flexion (PFL). While both feet of the subject were placed on a footplate that was connected to a torque meter and could be rotated by a motor, the subjects' arms were relaxed and kept on the lap during all measurements.

For $\mathrm{R}$ the footplate was fixed at the starting position for DFL and PFL conditions, as described above. During DFL and PFL, a sinusoidal force was applied synchronously to both feet (duration of increase of force $500 \mathrm{~ms}$ ), while recording was only taken from the right side. The position of the right footplate was displayed continuously on an optical scale for the subject, who was instructed to react to the induced force as fast as possible in order to keep the displacement of the footplate minimal. The maximal force applied was adjusted to be approximately $50 \%$ of the individual maximal force in $\mathrm{M}$. tibialis anterior.
4.3.2.2. Standing perturbations similar to BST. In a standing position, subjects were exposed to bilateral forward (PERf) and backward perturbations (PERb) with a dislocation of individually adjusted amplitudes $(13$ to $15 \mathrm{~cm}$ ) on a treadmill (Woodway). The acceleration of the translation was $2.8 \mathrm{~m} \mathrm{~s}^{2}$ - with a rise time of $500 \mathrm{~ms}$. The optimal standing position with minimal pre-activation in $\mathrm{M}$. tibialis anterior, $\mathrm{M}$. gastrocnemius medialis and $M$. soleus was individually determined at the beginning of each experiment. Differential force in the anteroposterior direction was measured by piezoelectric transducers in the treadmill and continuously displayed online on a screen in front of the subject in order to assist with the maintenance of the defined body position. The starting position was marked and subjects went back to this defined position after each dislocation. Subjects compensated the dislocation of the treadmill without flexing the knee or a step in any direction. If subject could not keep the balance, trails were rejected. Hands and arms were positioned on both sides beside the body.

\subsection{Statistics}

Group data are presented as mean values and standard errors of the mean ( $($ SEM). To test for significant differences between the measurements taken before and after training, an analysis of variance (ANOVA, repeated measurement design) was applied with factors "training" (SMT, BST, and CON) and "task" ( $R$, DFL, PFL, PERf and PERb) for the individual differences which were calculated between the assessment before training subtracted from that obtained after the training intervention (post-pre). For post-hoc analysis of first order effects, Fisher's PLSD was used. If ANOVA proved significant interaction effects of task and training, one sample analysis (hypothesized mean $=0$ ) was used to analyze which particular combination of training and task produced a significant difference between the data recorded before and after training. All analyses were executed using SAS StatView for Windows 5.0 software.

\section{Acknowledgments}

We thank Dr. Franz Aiple, Tom Günther, Florian Pfister and Frank Huethe for technical assistance. This work was supported by the Deutsche Forschungsgemeinschaft (SCHU 1487/1-2).

\section{REFERENCES}

Aagaard, P., Simonsen, E.B., Andersen, J.L., Magnusson, P., Dyhre-Poulsen, P., 2002. Increased rate of force development and neural drive of human skeletal muscle following resistance training. J. Appl. Physiol. 93, 1318-1326.

Adkins, D.L., Boychuk, J., Remple, M.S., Kleim, J.A., 2006. Motor training induces experience-specific patterns of plasticity across motor cortex and spinal cord. J. Appl. Physiol. 101, 1776-1782.

Bonato, C., Zanette, G., Polo, A., Bongiovanni, G., Manganotti, P., Tinazzi, M., Teatini, F., Fiaschi, A., 1994. Cortical output 
modulation after rapid repetitive movements. Ital. J. Neurol. Sci. 15, 489-494.

Bonato, C., Zanette, G., Fiaschi, A., Rossini, P.M., 2002. Activity-dependent modulation of synaptic transmission in the intact human motor cortex revealed with transcranial magnetic stimulation. Cereb. Cortex 12, 1057-1062.

Bonnard, M., Camus, M., Coyle, T., Pailhous, J., 2002. Task-induced modulation of motor evoked potentials in upper-leg muscles during human gait: a TMS study. Eur. J. Neurosci. 16, 2225-2230.

Butefisch, C.M., Khurana, V., Kopylev, L., Cohen, L.G., 2004. Enhancing encoding of a motor memory in the primary motor cortex by cortical stimulation. J. Neurophysiol. 91 , 2110-2116.

Camus, M., Pailhous, J., Bonnard, M., 2004. Cognitive tuning of corticospinal excitability during human gait: adaptation to the phase: Eur. J. Neurosci. 20, 1101-1107.

Carroll, T.J., Riek, S., Carson, R.G., 2002. The sites of neural adaptation induced by resistance training in humans. J. Physiol. 544, 641-652.

Chen, R., Tam, A., Butefisch, C., Corwell, B., Ziemann, U., Rothwell, J.C., Cohen, L.G., 1998. Intracortical inhibition and facilitation in different representations of the human motor cortex. J. Neurophysiol. 80, 2870-2881.

Christensen, L.O.D., Andersen, J.B., Sinkjar, T., Nielsen, J., 2001. Transcranial magnetic stimulation and stretch reflexes in the tibialis anterior muscle during human walking. J. Physiol. (Lond) 531, 545-557.

Classen, J., Liepert, J., Wise, S.P., Hallett, M., Cohen, L.G., 1998. Rapid plasticity of human cortical movement representation induced by practice. J. Neurophysiol. 79, 1117-1123.

Darling, W.G., Wolf, S.L., Butler, A.J., 2006. Variability of motor potentials evoked by transcranial magnetic stimulation depends on muscle activation. Exp. Brain Res. 174 376-385.

Devanne, H., Lavoie, B.A., Capaday, C., 1997. Input-output properties and gain changes in the human corticospinal pathway. Exp. Brain Res. 114, 329-338.

Doyon, J., Benali, H., 2005. Reorganization and plasticity in the adult brain during learning of motor skills. Curr. Opin. Neurobiol. 15, 161-167.

Duchateau, J., Hainaut, K., 1984. Isometric or dynamic training: differential effects on mechanical properties of a human muscle. J. Appl. Physiol. 56, 296-301.

Enoka, R.M., 1997. Neural adaptations with chronic physical activity. J. Biomech. 30, 447-455.

Floyer-Lea, A., Matthews, P.M., 2004. Changing brain networks for visuomotor control with increased movement automaticity. J. Neurophysiol. 92, 2405-2412.

Gruber, M., Gruber, S.B., Taube, W., Schubert, M., Beck, S.C., Gollhofer, A., 2007a. Differential effects of ballistic versus sensorimotor training on rate of force development and neural activation in humans. J. Strength Cond. Res. 21, 274-282.

Gruber, M., Taube, W., Gollhofer, A., Beck, S., Amtage, F., Schubert, M., 2007b. Training-specific adaptations of $\mathrm{H}$ - and stretch reflexes in human soleus muscle. J. Mot. Behav. 39, 68-78.

Jacobs, K.M., Donoghue, J.P., 1991. Reshaping the cortical motor map by unmasking latent intracortical connections. Science 251, 944-947.

Jensen, J.L., Marstrand, P.C., Nielsen, J.B., 2005. Motor skill training and strength training are associated with different plastic changes in the central nervous system. J. Appl. Physiol. 99, 1558-1568.

Komi, P.V., 1986. Training of muscle strength and power: interaction of neuromotoric, hypertrophic, and mechanical factors. Int. J. Sports Med. 7 (Suppl 1), 10-15.

Kujirai, T., Caramia, M.D., Rothwell, J.C., Day, B.L., Thompson, P.D., Ferbert, A., Wroe, S., Asselman, P., Marsden, C.D., 1993.
Corticocortical inhibition in human motor cortex. J. Physiol. $471,501-519$.

Liepert, J., Miltner, W.H., Bauder, H., Sommer, M., Dettmers, C., Taub, E., Weiller, C., 1998. Motor cortex plasticity during constraint-induced movement therapy in stroke patients. Neurosci. Lett. 250, 5-8.

Lotze, M., Braun, C., Birbaumer, N., Anders, S., Cohen, L.G., 2003. Motor learning elicited by voluntary drive. Brain 126 , 866-872.

Muellbacher, W., Ziemann, U., Boroojerdi, B., Cohen, L., Hallett, M., 2001. Role of the human motor cortex in rapid motor learning. Exp. Brain Res. 136, 431-438.

Muellbacher, W., Ziemann, U., Wissel, J., Dang, N., Kofler, M., Facchini, S., Boroojerdi, B., Poewe, W., Hallett, M., 2002. Early consolidation in human primary motor cortex. Nature 415 , 640-644.

Pascual-Leone, A., Grafman, J., Hallett, M., 1994. Modulation of cortical motor output maps during development of implicit and explicit knowledge. Science 263, 1287-1289.

Pascual-Leone, A., Nguyet, D., Cohen, L.G., Brasil-Neto, J.P., Cammarota, A., Hallett, M., 1995. Modulation of muscle responses evoked by transcranial magnetic stimulation during the acquisition of new fine motor skills. J. Neurophysiol. 74, 1037-1045.

Perez, M.A., Lungholt, B.K., Nyborg, K., Nielsen, J.B., 2004. Motor skill training induces changes in the excitability of the leg cortical area in healthy humans. Exp. Brain Res. 159, 197-205.

Perez, M.A., Lundbye-Jensen, J., Nielsen, J.B., 2006. Changes in corticospinal drive to spinal motoneurones following visuo-motor skill leaming in humans. J. Physiol. 573, 843-855.

Ploutz, L.L., Tesch, P.A., Biro, R.L., Dudley, G.A., 1994. Effect of resistance training on muscle use during exercise. J. Appl. Physiol. 76, 1675-1681.

Puttemans, V., Wenderoth, N., Swinnen, S.P., 2005. Changes in brain activation during the acquisition of a multifrequency bimanual coordination task: from the cognitive stage to advanced levels of automaticity. J. Neurosci. 25, $4270-4278$.

Schmidtbleicher, D., Haralambie, G., 1981. Changes in contractile properties of muscle after strength training in man. Eur. J. Appl Physiol. Occup. Physiol. 46, 221-228.

Schneider, C., Devanne, H., Lavoie, B.A., Capaday, C., 2002. Neural mechanisms involved in the functional linking of motor cortical points. Exp. Brain Res. 146, 86-94.

Schubert, M., Curt, A., Jensen, L., Dietz, V., 1997. Corticospinal input in human gait: modulation of magnetically evoked motor responses. Exp. Brain Res. 115, 234-246.

Schubert, M., Curt, A., Colombo, G., Berger, W., Dietz, V., 1999. Voluntary control of human gait: conditioning of magnetically evoked motor responses in a precision stepping task. Exp. Brain Res. 126, 583-588.

Taube, W., Schubert, M., Gruber, M., Beck, S., Faist, M., Gollhofer, A., 2006. Direct corticospinal pathways contribute to neuromuscular control of perturbed stance. J. Appl. Physiol. $101,420-429$.

Taube, W., Gruber, M., Beck, S., Faist, M., Gollhofer, A., Schubert, M., 2007. Cortical and spinal adaptations induced by balance training: correlation between stance stability and corticospinal activation. Acta Physiol. (Oxf) 189, 347-358.

Van Cutsem, M., Duchateau, J., Hainaut, K., 1998. Changes in single motor unit behaviour contribute to the increase in contraction speed after dynamic training in humans. J. Physiol. 513, 295-305.

Voigt, M., Chelli, F., Frigo, C., 1998. Changes in the excitability of soleus muscle short latency stretch reflexes during human hopping after 4 weeks of hopping training. Eur. J. Appl. Physiol. Occup. Physiol. 78, 522-532. 
Werhahn, K.J., Kunesch, E., Noachtar, S., Benecke, R., Classen, J., 1999. Differential effects on motor cortical inhibition induced by blockade of GABA uptake in humans. J. Physiol. 517 (Pt 2), 591-597.

Wu, T., Kansaku, K., Hallett, M., 2004. How self-initiated memorized movements become automatic: a functional MRI study. J. Neurophysiol. 91, 1690-1698.

Zehr, P.E., 2002. Considerations for use of the Hoffmann reflex in exercise studies. Eur. J. Appl. Physiol. 86, $455-468$ 